\title{
A Prediction Method for Galloping of Transmission Lines Based on Improved Neural Network
}

\section{Yongfeng Cheng, Jingshan Han*, Bin Liu, Danyu Li}

China Electric Power Research Institute, Beijing, China

Email address:

jenkinhan@163.com (Jingshan Han)

${ }^{*}$ Corresponding author

\section{To cite this article:}

Yongfeng Cheng, Jingshan Han, Bin Liu, Danyu Li. A Prediction Method for Galloping of Transmission Lines Based on Improved Neural Network. International Journal of Mechanical Engineering and Applicationss. Vol. 6, No. 4, 2018, pp. 126-133.

doi: 10.11648/j.ijmea.20180604.15

Received: September 21, 2018; Accepted: October 31, 2018; Published: November 8, 2018

\begin{abstract}
The traditional back-propagation neural network (BP) have the disadvantages including the random generation of initial weights and thresholds, easy to fall into the local optimization, and the convergence speed is slow, and it's hard to confirm the number of neurons in hidden layer. In this paper, the Genetic Algorithm (GA) is utilized to optimize the initial weights and thresholds space of the BP neural network. To obtain the optimal weight matrix and threshold matrix, the error-forward-feedback neural network training is carried out by using the data of transmission line galloping. The trial and error method are used to reduce the number of hidden layer neurons and find the optimal number of neurons. An optimized GA-BP neural network model is established to warn the occurrence of transmission line galloping. The historical data of the transmission lines galloping in the related areas is analyzed by the optimized GA-BP neural network model. The validity and practicability of the proposed GA-BP neural network model is tested and verified. The simulation results show that the GA-BP neural network module could predict the galloping situation of transmission lines more accurately and effectively. As a result, it provides a strong guarantee for preventing large-scale grid fault disasters, and further improves the power grid's ability to withstand natural disasters.
\end{abstract}

Keywords: Genetic Algorithm, BP Neural Network, Machine Learning Method, Hidden Layer, Transmission Line Galloping

\section{Introduction}

The transmission lines galloping is one of the common electric faults in power grid. It is a phenomenon of galloping with low frequency and large amplitude caused by continuous wind load under the condition of uneven icing [1]. One of the important characteristics of conductor galloping is that it is very destructive. It is easy to cause flashover, tripping, damage to fittings, insulator breakage, conductor strand breakage and even tower collapse and other serious power accidents, resulting in a large area of blackout, which seriously threatens society. The transmission lines network is spread all over the country, the location and climate conditions are particularly complex. In autumn, winter and spring, transmission lines are vulnerable to strong wind, ice and snow weather, causing large-scale conductor galloping. The frequency of bad weather caused by climate change increases year by year, and the probability, the scope and extent of conductor galloping disasters are also increased [2, 3, 4]. Therefore, it is of great significance to improve the warning level of conductor galloping to ensure the safe and stable operation of power system.

At present, a lot of research work has been done in the prevention and early warning of transmission lines galloping at home and abroad. In reference [5], a method which using computer simulation technology and combining with real-time meteorological conditions to simulate wire galloping was proposed, which can establish real-time model according to preset conditions, but cannot consider the influence of geographical location, humidity and other major factors on wire galloping. In literature [6], the author proposed to use unified wireless communication transmission technology, sensor technology and cloud data technology to deal with on-line monitoring problems on $1000 \mathrm{KV}$ transmission lines, 
including the application of on-line monitoring and management device of conductor galloping in engineering, to achieve real-time data collection and processing and comprehensive analysis, but there are still unavoidable problems: limited monitoring range, unstable transmission signal, high cost, difficult maintenance, etc., cannot fully meet the requirements of practical application. In reference [7], to predict the wire galloping, an icing prediction model based on data-driven algorithm and least squares support vector machine is proposed, which can predict the icing thickness in advance according to the meteorological conditions. In reference [8], a prediction method by using BP neural network was proposed. BP neural network is a kind of feedforward neural network with multi-layer structure. It has forward propagation, error back propagation and continuous transfer function. It uses the steepest gradient descent method and error back propagation algorithm. Through back propagation, the weights and thresholds are adjusted continuously to minimize the sum of squares of errors. However, in practical engineering applications, it is found that BP algorithm has the following shortcomings [9]: (1) The optimal number of neurons in the hidden layer is not clearly defined; (2) BP neural network input initial weights and threshold randomness is large; (3) Gradient descent method is easy to fall into local optimal solution.

Therefore, from the actual needs, we need a strong adaptability, wide range of adaptability, strong initiative method to predict the wire galloping in time, to provide accurate early warning information and decision-making basis to avoid great loss.

This paper starts from the analysis of the galloping mechanism of transmission lines, and combined with the recorded data, synthetically analyzed the influence of various factors on the galloping state of transmission line, confirm the main related factors affecting the galloping of transmission line, and introduces the transmission line spacing into the algorithm for the first time. Based on the combination of BP neural network and genetic algorithms, according to the different theories of determining the number of hidden layer nodes, the optimal number range of neurons is obtained. On this basis, the optimal number of neurons is determined by trial and error method. A GA-BP neural network model is established to realize the early warning of transmission line galloping. Then taking the galloping data in different regions as an example to make an analysis, and compared with other machine learning algorithms. The results show that the proposed method is effective and practical.

\section{The Mechanism of Optimizing BP Neural Network}

\subsection{Optimize the Number of Nodes in Hidden Layer}

The number of neurons in hidden layer is directly related to the number of input and output nodes [10]. If the number of neurons is too large, the processing time and training time of neural network will increase, which may result in the decrease of generalization ability and fault tolerance. Therefore, a way which first determines the upper and lower limits of the optimal number of hidden layer neurons according to the three formulas which are widely used and relatively high accuracy, and verifies the optimal number in this range. By comparing the convergence speed, training error and test error of the network, the optimal number of neurons can be obtained as far as possible and avoided the shortage of traditional random settings, while increasing accuracy, it also reduces unnecessary computation [11].

\subsection{Mechanism of BP Neural Network Improved by Genetic Algorithm}

Genetic Algorithm (GA) is a method of searching the optimal solution by simulating the natural selection and genetic mechanism of Darwin's biological evolution. It has the characteristics of randomness, high efficiency, parallelism and global optimization [12]. First, genetic algorithm does not use the traditional gradient descent algorithm, but imitates the natural evolution process to search for the global optimal solution. Second, genetic algorithm is different from the traditional random search method. Genetic algorithm searches based on making full use of historical information [13]. Therefore, genetic algorithm is used to improve the process which get initial weights and thresholds randomly, so that the neural network can obtain the optimal weights and thresholds, to avoid the neural network get into the local optimal solution defects, and improve the accuracy of prediction.

\section{Establish an Optimized Model}

\subsection{Optimization of Input Layer for BP Neural Network}

The topology of BP neural network is divided into three layers: input layer, hidden layer and output layer. According to the transverse wind dance mechanism proposed by Den Hartog [14], torsional dance theory proposed by Nigol [15] and conductor dance model proposed by Blevins and Iwan [16], it is found that the galloping of transmission lines is the direct or indirect result of many factors.

\subsubsection{The Influence of the Transmission Line Structure}

In reference [17], it suggests that the span is an important factor in causing conductor galloping. Under the same material, the shorter the span is, the greater the relative torsional stiffness of the conductor is, and the less likely it is to torsion. Under the action of transverse wind, eccentric airfoil icing is easily formed, the greater the lift is, and the greater the possibility of galloping is. At the same time, it is found by the article that there is no definite relationship between different voltage levels and the conductor galloping over the years.

\subsubsection{The Influence of Conductor Icing}

In reference [18], by simulating the occurrence of conductor galloping, under the simultaneous action of certain humidity, temperature and wind speed, it is proposed that an 
inhomogeneous layer of ice on the conductor surface which changes the cross-section shape and gravity of the conductor itself, thus causing torsion under the action of gravity itself. It is one of the most important conditions for causing the conductor galloping.

\subsubsection{The Influence of Meteorological Factors}

In literature [19], it suggests that conductor galloping occurs only when the conductor is subjected to wind excitation after icing. Therefore, various meteorological factors causing conductor icing are also necessary conditions for conductor galloping, including temperature, humidity, precipitation, wind direction and wind speed. Through literature research and historical dance data research, the occurrence of dance disasters is generally concentrated in the

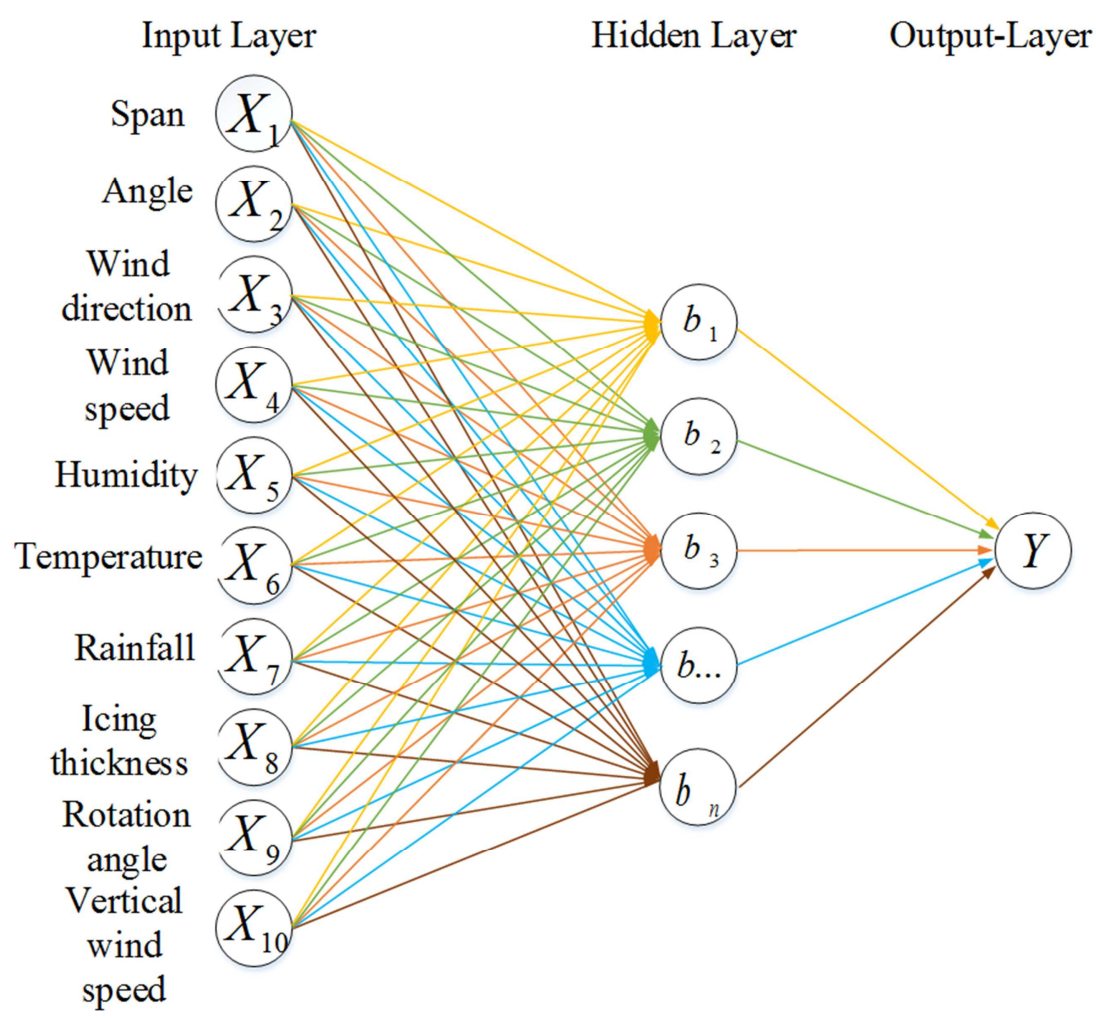

Figure 1. The model of transmission line galloping by BP neural network. first, second, third, fourth, tenth, eleventh and twelfth months, which mainly concentrated in rain and snow weather, the temperature is mainly between $-5^{\circ} \mathrm{C}$ and $3^{\circ} \mathrm{C}$ There is a certain angle between the conductor and the wind direction.

Therefore, to predict the galloping results more accurately and enhance the generalization ability of the model, this paper enlarges the data input dimension, adds the transmission line spacing and vertical wind speed into the input layer, and uses 10 influence factors as the input parameters of the input layer, including the line spacing, line angle, wind speed, wind direction, humidity, temperature. Rainfall, ice thickness, angle, vertical wind speed, the output layer for the prediction results, that is, whether the conductor will gallop, draw BP neural network as shown in Figure 1.

\section{Hidden Layer Output-Layer}


(2) Take the integer as the number of nodes and input it into the BP neural network optimized by genetic algorithm for training.

(3) Record the convergence speed and training accuracy of neural network training which corresponds to per nodes.

\subsection{Constructing a BP Neural Network Optimized by Genetic Algorithm}

According to the principle of genetic algorithm optimizing BP neural network [20], aiming at overcoming the shortcomings of BP neural network, such as the randomness of initial weights and thresholds, GA-BP neural network is modeled according to the following steps.

Step one: chromosome coding, constructing the initial population.

By using real number encoding or binary encoding the weights and threshold to encode-string, then encoding to chromosome. In this paper, a BP neural network with three-layer topology is constructed. The number of neurons in input layer, hidden layer and output layer is assumed to be $i$ 、 $k 、 j$, and the corresponding weights of hidden layer and output layer are assumed to be $W_{1} 、 W_{2}$, and the thresholds are $B_{1}, B_{2}$. The initial length of gene string is: $i \times k+k \times j+k+j$. The weights and thresholds correspond to the locations of the genes in the gene strand, producing the initial population synthesized by $M$ chromosomes.

Step two: select the individual evaluation function.

Individual fitness determines the probability of inheritance to the next generation. The reciprocal of the mean square error is defined as fitness function. When the mean square error reaches the minimum, the optimal weight and threshold are obtained. The fitness function is defined as follows:

$$
f(i)=\frac{1}{M S E_{i}}=\frac{N}{\sum(A-T)^{2}}
$$

In the formula, $f(i)$ Represents the fitness value of the chromosome.

Step three: selection, crossover, mutation operation.

Arranged according to the size of individual fitness values, from 1 to $\mathrm{M}$, according to the proportion of chromosome fitness, roulette method was used to calculate the probability of individual selection. The real number crossover method is also chosen according to the real number coding mechanism. The crossover of the $k$ gene $w_{k}$ and the $l$ gene $w_{l}$ at $j$ level are:

$$
\begin{aligned}
& w_{k j}=w_{k j}(1-b)+w_{l j} b \\
& w_{l j}=w_{l j}(1-b)+w_{k j} b
\end{aligned}
$$

$b$ is the data obtained randomly from $[0,1]$.

The value of some genes in the chromosome coding string is exchanged to produce a new chromosome. The $j$ gene of the $i$ individual is operated according to the principle of uniform variation, as follows:

$$
\begin{gathered}
w_{i j}=\left\{\begin{array}{c}
w_{i j}+\left(w_{i j}-w_{\max }\right) f(g), \quad r \geq 0.5 \\
w_{i j}+\left(w_{\min }-w_{i j}\right) f(g), \quad r<0.5
\end{array}\right. \\
f(g)=r_{2}\left(1-g / G_{\max }\right)
\end{gathered}
$$

$w_{\max }$ and $w_{\min }$ represent the upper and lower bounds of gene values, $\mathrm{C}$ is a random number between $[0,1], \mathrm{D}$ represents the current number of iterations, f represents the largest evolutionary algebra.

Step four: determine genetic algebra.

According to the fitness value of chromosome, the individual with the best fitness value is selected. With the continuous progress of genetic algorithm, the evaluation function will reach a stable value, and the genetic algebra $N$ is recorded at this time. The weights and thresholds of the final output are used as the initial weights and thresholds of the BP neural network.

Step five: create a neural network

The contents of input and output layers have been determined according to the influencing factors and prediction results of conductor galloping. Because there are many complicated factors affecting conductor galloping and the nonlinear relationship is prominent, it is necessary to select an activation function which can better fit the nonlinear relationship. The activation functions mainly include tanh, sigmoid, ReLU and so on. Traditional neural network uses sigmod function as activation function, which has the following drawbacks: firstly, the weight has little effect on loss function, resulting in gradient dispersion; secondly, the output of function is not centered on zero, resulting in the reduction of weight update efficiency [21].

$$
f(x)=\left\{\begin{array}{l}
0, x \leq 0 \\
x, x>0
\end{array}\right.
$$

Therefore, the linear rectification function ReLU is chosen as the activation function. As shown in formula (7), its converge much faster than sigmoid and tanh functions, which can effectively alleviate the problem of gradient disappearance.

Step six: setting up network learning efficiency

Learning efficiency determines whether the neural network can converge and the speed of convergence, if learning efficiency is set too large lead to convergence too fast, cannot get the optimal value; if were set too small, will lead to the neural network into the local optimal solution limitations. The function ReLU requires a strict learning rate. After many experiments, the learning rate is chosen to be 3 . The training accuracy needs to be adjusted continuously through each experiment to complete the neural network configuration and training.

Step seven: train and test the generalization ability of the optimized BP neural network.

The pre-processing samples are input into the model, and the network parameters are finely tuned through the 
comparison results. Input 1000 groups of test samples, and finally get the dance prediction results, the prediction results and the actual dancing situation are compared, calculate the correct rate, judge the reliability of the dance early warning model.

\section{Prediction Examples of Galloping Based on Improved GA-BP Neural Network}

The data of transmission line galloping used in this paper comes from the galloping warning center database of China Electric Power Research Institute, Hubei, Henan and Hunan Provincial Company of State Grid. Using MATLAB to optimize the genetic algorithm combined with BP neural network algorithm programming, this paper randomly selected 5000 data in the database, contains 400 piece of wire galloping data. 4000 pieces of data are extracted as training sets, including 320 pieces of dancing data, the remaining 1000 pieces of data are used as model test sets. The training times vary from 500 to 5000 . At the same time, support vector machine algorithm, Adaboost algorithm and traditional BP neural network algorithm are used to train and predict the same group of data and optimize them. The prediction accuracy of GA-BP neural network is compared. To make better use of the neural network principle to predict, the output results are expressed by 0 or 1,0 represents no dance, 1 represents a dance.

Because the data of transmission lines galloping are collected widely, and the ways of data preservation are inconsistent, it is necessary to pre-process the data. It mainly includes four parts: data-cleaning, data-integration, data-transformation and data-protocol. Firstly, multiple data sources are put into a unified data warehouse, then erroneous data are deleted, then Lagrange interpolation is used to interpolate the missing data. Finally, the data is normalized to balance the impact on the network and reduce the error. 10 sets of examples are taken out from training data set, as shown in Table 1.

Table 1. Input parameter of the train samples.

\begin{tabular}{|c|c|c|c|c|c|c|c|c|c|c|c|}
\hline ID & Span & Angle & $\begin{array}{l}\text { Wind } \\
\text { direction }\end{array}$ & Wind speed & Humidity & Temperature & Rainfall & Icing thickness & Rotation angle & $\begin{array}{l}\text { Vertical } \\
\text { speed }\end{array}$ & Galloping \\
\hline 1 & 301 & 296.35 & 64.47 & 13.13 & 0.90 & -0.77 & 0.20 & 0.4434 & 1.88 & 2.3296 & 1 \\
\hline 2 & 361 & 236.53 & 81.22 & 11.54 & 0.93 & -0.85 & 0.26 & 0.4921 & 24.69 & 0.7074 & 1 \\
\hline 3 & 527 & 307.62 & 99.98 & 6.01 & 0.89 & 0.62 & 0.28 & 0.3011 & 27.64 & 2.7881 & 1 \\
\hline 4 & 169 & 248.70 & 87.74 & 8.68 & 0.92 & 0.24 & 0.25 & 0.3684 & 19.04 & 2.8317 & 1 \\
\hline 5 & 277 & 186.49 & 347.88 & 2.22 & 0.94 & 1.25 & 0.15 & 0.0979 & 18.61 & 0.7084 & 1 \\
\hline 6 & 407 & 213.28 & 55.07 & 2.51 & 0.65 & -0.19 & 0.00 & 0.0000 & 21.79 & 0.9317 & 0 \\
\hline 7 & 204 & 233.16 & 87.75 & 3.3 & 0.99 & 6.80 & 1.18 & 0.0000 & 34.59 & 1.8734 & 0 \\
\hline 8 & 370 & 150.21 & 25.51 & 3.06 & 0.98 & -0.19 & 0.15 & 0.1118 & 55.30 & 2.5158 & 0 \\
\hline 9 & 805 & 163.10 & 321.95 & 1.96 & 0.87 & 3.31 & 0.18 & 0.0000 & 21.06 & 0.7042 & 0 \\
\hline 10 & 662 & 248.49 & 332.60 & 1.73 & 0.90 & 3.99 & 0.19 & 0.0000 & 84.11 & 1.7209 & 0 \\
\hline
\end{tabular}

According to the formula (1), (2), (3), the upper and lower bounds of the optimal number of hidden layer neurons are finally taken $n_{1 \_\max }=10, n_{1 \_ \text {min }}=3$. In this range, the BP neural network optimized by genetic algorithm is introduced to calculate the corresponding training errors and test errors. The results are shown in Table 2.

Table 2. The relationship between the number ofhidden layer units and error.

\begin{tabular}{lll}
\hline hidden-layer neurons & training errors & test errors \\
\hline 3 & 0.0178 & 0.0233 \\
4 & 0.0199 & 0.0209 \\
5 & 0.0112 & 0.0102 \\
6 & 0.0162 & 0.0195 \\
7 & 0.0177 & 0.0178 \\
8 & 0.0217 & 0.0342 \\
9 & 0.0188 & 0.0298 \\
10 & 0.0157 & 0.0203 \\
\hline
\end{tabular}

According to the above table, the curves of the number of hidden layer nodes and the prediction error of the neural network are drawn, as shown in Figure 2.

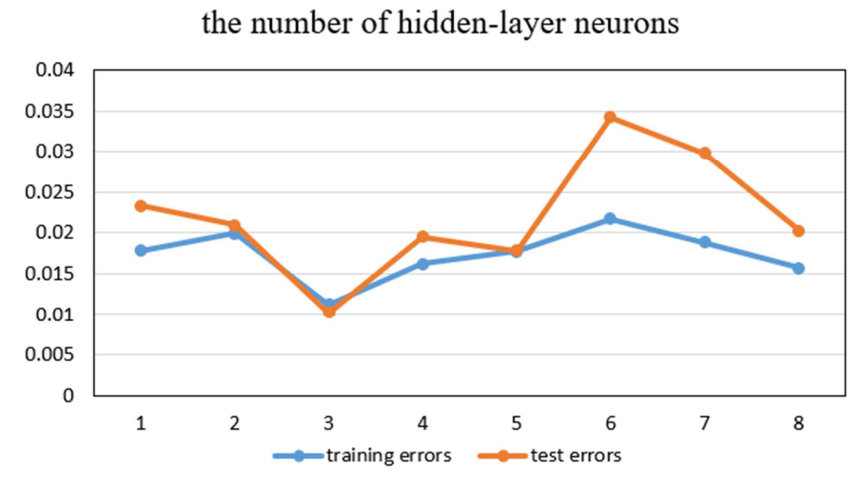

Figure 2. Error curve for the relationship between the number of hidden layer units and error.

It can be clearly found that in the range of the optimal number of neurons, with the number of nodes changing from small to large, the accuracy of the prediction results of the whole neural network also changes. When the number of hidden layer neurons is 5 , the training error and the test error both reach the minimum, and reach the ideal state in the range. At the same time, it is found that When the number of neurons is 10 , the prediction error is relatively small, but as the number 
of hidden layer neurons increases, on the one hand, the training time will increase, on the other hand, the generalization ability of the model may decline, so five neuron nodes are used to input into the model, and the model is used to train again.

Best Validation Performance is 0.0034549 at epoch 15

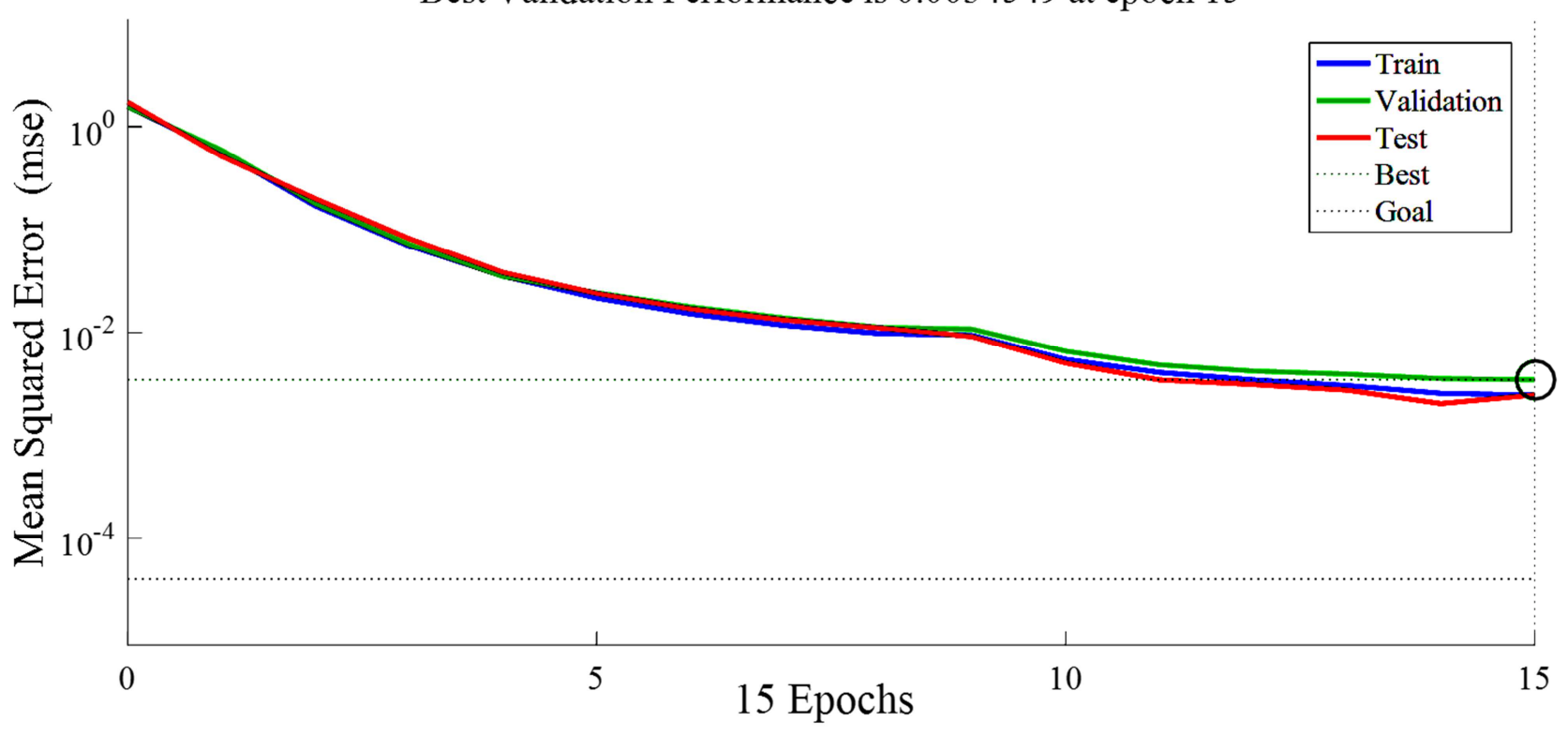

Figure 3. Error curve for the optimization of the weights and thresholds.

From the mean square error curve in Figure 3, it can be seen that the training errors of the optimal weights and thresholds decrease with the increase of iterations. When the number of iterations is 15 , the mean square error stabilizes below 0.01 , and no longer decreases with the increase of genetic algebra. Currently, the weights and thresholds are optimal values. In the neural network, the improved GA-BP neural network is used to compare the predicted values of 1000 groups of wire galloping test samples with the real values. As shown in Figure 4, 100 groups of samples are shown. The abscissa is the serial number of the test samples, and the ordinate is the predicted output value and the expected value of the neural network.

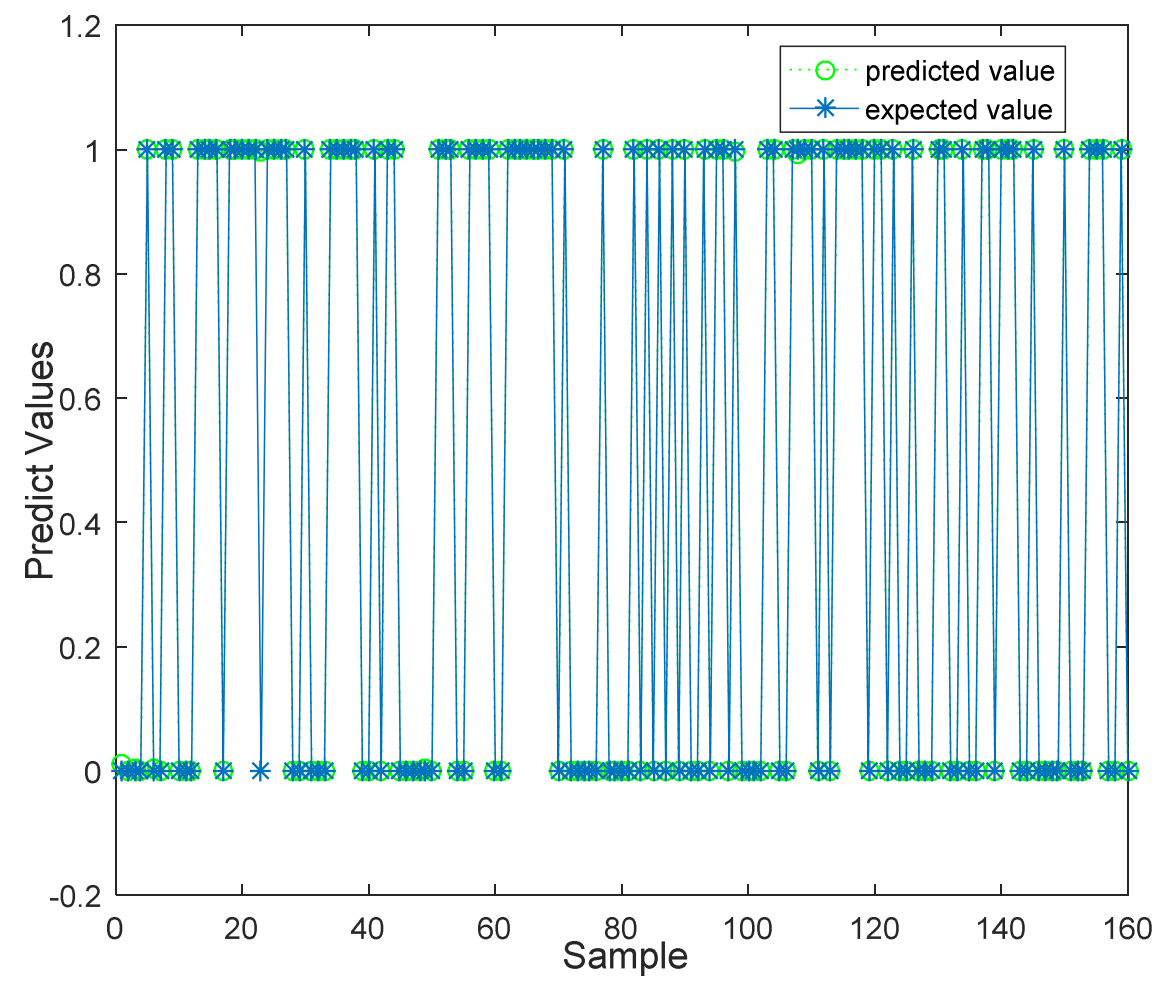

Figure 4. Contrasts between the true value and the predictive value of GA-BP neural network. 
Statistical analysis of the predicted output results shows that the predict accuracy of the optimized GA-BP neural network model can reach at $99.96 \%$, and the standard deviation reach at $0.32 \%$.

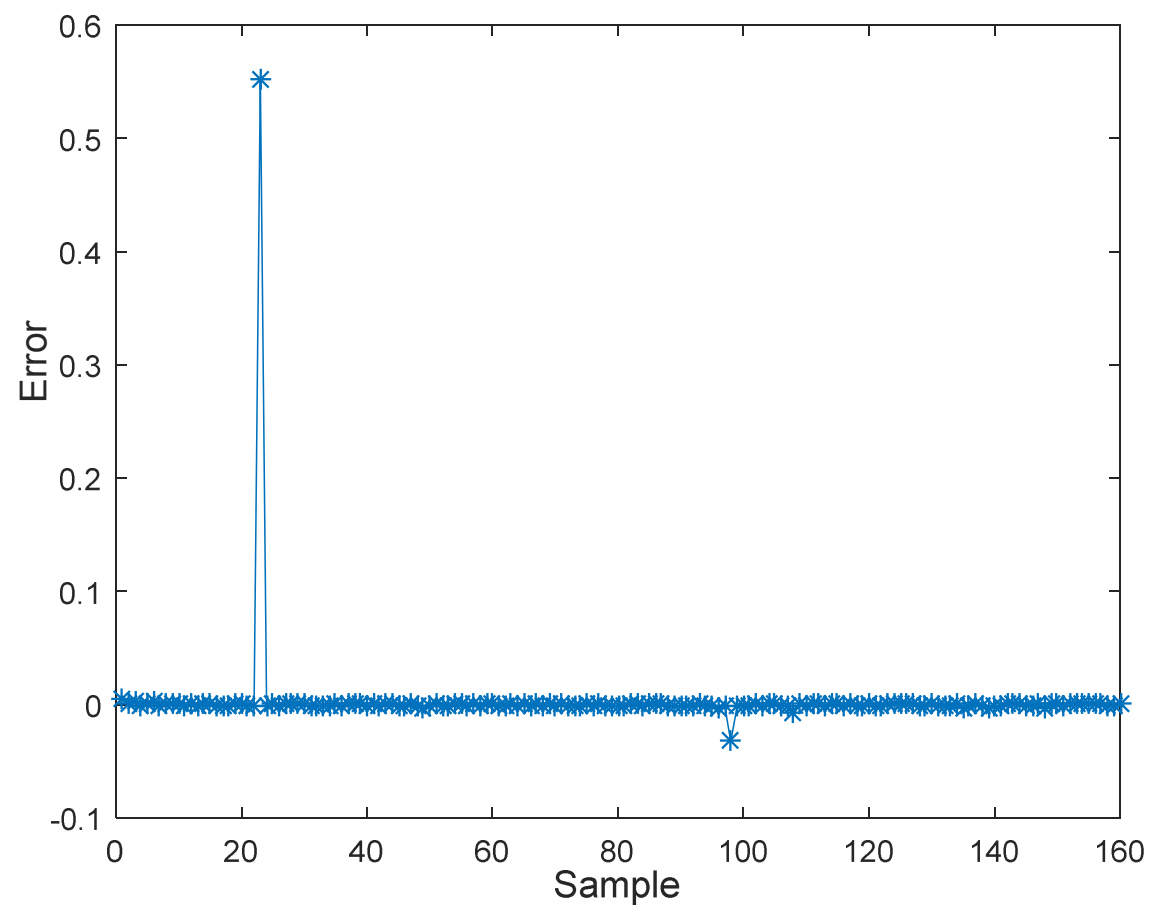

Figure 5. The error of predictive value by GA-BP neural network.

In the process of forecasting, there is a certain error anomaly, such as the sample with prediction error of 0.1 in Figure 5, the prediction error is larger than other samples. The reasons are mainly in two aspects: First, considering the nature of the algorithm itself, because the BP neural network optimized by genetic algorithm is ultimately to have a greater possibility of obtaining the global maximum. Optimal solution is still a probabilistic problem, for the whole sample, there will be a higher and more accurate prediction accuracy, but at the same time there may be an individual, in probability cannot get the optimal solution, resulting in greater errors; second, because the transmission line galloping in power grid disaster accidents has been a difficult problem, whether it is forecasting technology or anti-dancing technology, the acquisition and recording of data are mostly obtained manually under adverse weather conditions, or after the occurrence of dancing disasters, the relevant personnel record the dancing occurrence according to historical meteorological forecast data, due to subjective factors and reasons, as a result, the data itself will also have some problems.

Table 3. The comparison of prediction by different algorithm.

\begin{tabular}{|c|c|c|c|c|}
\hline algorithm & Improved GA-BP neural network & BP neural network & SVM & Adaboost algorithm \\
\hline Accuracy of training set & $99.98 \%$ & $94.54 \%$ & $86.37 \%$ & $90.21 \%$ \\
\hline Accuracy of testing set & $99.96 \%$ & $93.88 \%$ & $82.14 \%$ & $88.10 \%$ \\
\hline
\end{tabular}

According to the principle of different algorithms in Table 3, the model is built with MATLAB, the training samples are trained with the same set of pre-processed training samples, and the testing samples are tested with the same set of testing samples. The training sets and testing sets corresponding to each algorithm are sorted out. As shown in Table 3, several different machine learning algorithms are compared and optimized. GA-BP neural network has a relatively good performance in the training set and test set, the highest accuracy is $12 \%$ higher than other algorithms, and found that its operation is faster, more efficient, and more stable.

At the same time, because of the characteristics of neural network is different from the traditional support vector machine algorithm, with the training data and the number of training increases, the accuracy of model prediction results will continue to improve, and ultimately achieve the desired state. Figure 3 to 5 and related data also show that the optimized genetic algorithm combined with BP neural network for transmission line galloping early warning has greater development prospects and stability.

\section{Conclusion}

According to the principle of traditional machine learning algorithm, this paper proposes and validates the optimized transmission line galloping warning algorithm (GA-BP) which based on genetic algorithm and BP neural network. 10 factors related to transmission line galloping are added as input elements to the BP neural network model optimized by improved genetic algorithm for the first time, and the 
generalization ability is enhanced. At the same time, the number of hidden layer neurons is optimized to avoid the disadvantage of traditional algorithm randomly selecting the number of nodes; The feature of searching global optimal solution by genetic algorithm is fully utilized to overcome the disadvantage that BP neural network is easy to fall into local optimal solution, which makes the prediction result more stable. The prediction accuracy is significantly higher than that of the conventional machine learning algorithm model. The application of the optimized GA-BP neural network model in the galloping early warning of transmission lines is fully proved that it is scientific and effective.

\section{Acknowledgements}

This work is supported by The National Natural Science Foundation of China (Grant No. 51678548)

\section{References}

[1] GUO Yinglong, Yan Li, Bao Wujun, et al. Research on Transmission Wire Galloping [J]. Journal of Wuhan University (Engineering Science), 1995 (5): 506-509.

[2] LIANG Zhifeng. Statistical Analysis of Transmission Trips of Transmission Lines of State Grid Corporation of China from 2011 to 2013 [J]. East China Electric Power, 2014, 42 (11): 2265-2270.

[3] ZHU Kuanjun, Liu Bin, Liu Chaoqun, et al. Anti-dancing research of UHV transmission lines [J]. High Voltage Engineering, 2007, 33 (11): 12-20.

[4] HUANG Xinbo, Sun Qindong, Cheng Ronggui, et al. Mechanical Analysis of Wire Icing and Online Monitoring System for Ice Coating [J]. Automation of Electric Power Systems, 2007, 31 (14): 98-101.

[5] YU Junqing, Guo Yinglong, Xiao Xiaohui. Computer Simulation of Transmission Wire Galloping [J]. Journal of Wuhan University (Engineering Science), 2002, 35 (1): 39-43.

[6] LIU Hongwei, Li Junfeng, Wang Changfei. Application of Online Monitoring and Management Platform for $1000 \mathrm{kV}$ UHV Transmission Lines [J]. Automation of Electric Power Systems, 2009, 33 (23): 98-102.

[7] HUANG Yining, Xu Jiahao, Yang Chengshun, et al. Prediction of Transmission Line Ice Coating Based on Data Driven Algorithm and LS-SVM [J]. Automation of Electric Power Systems, 2014, 38 (15): 81-86.

[8] LIAO Wei, Xiong Xiaofu, Li Xin, et al. Early warning method of transmission line galloping based on BP neural network [J]. Power System Protection and Control, 2017, 45 (19): 154-161.

[9] YANG Wei, Ni Yidong, Wu Junji. Research on Initial Value and Convergence of Weights in BP Neural Networks [J]. Journal of Electric Power Systems and Automation, 2002, 14 (1): 20-22.

[10] SHEN Huayu, Wang Zhaoxia, Gao Chengyao, et al. Determination of the number of hidden layer elements in BP neural network [J]. Journal of Tianjin University of Technology, 2008, 24 (5): 13-15.

[11] SHI Kunpeng, Qiao Ying, Zhao Wei, et al. Short-term wind power prediction based on historical data entropy-related information mining [J]. Automation of Electric Power Systems, 2017, 41 (3): 13-18.

[12] ZHAI Wenxiang, Wang Wentian. Genetic Algorithm and Its Application [J]. Systems Engineering and Electronics, 1998, 23 (7): 9-10.

[13] TIAN Xuguang, Song Wei, Liu Yuxin. Optimization of BP neural network structure and parameters by genetic algorithm [J]. Journal of Computer Applications and Software, 2004, 21 (6): 69-71.

[14] Den Hartog J P. Transmission line vibration due to sleet [J]. Electrical Engineering, 2013, 51 (6):413-413.

[15] Nigol O, Clarke G J, Nigol O, et al. CONDUCTOR GALLOPING AND CONTROL BASED ON TORSIONAL MECHANISM [J]. IEEE Transactions on Power Apparatus \& Systems, 1974, PA93 (6):1729-1729.

[16] Blevins RD. The Galloping Response of a Two-Degree-of-Freedom System [J]. Journal of Applied Mechanics, 1974, 41 (4):1113.

[17] HUANG Jingya. Analysis and Research on Conductor Galloping of Overhead Transmission Lines [J]. China Electric Power, 1995 (2): 21-26.

[18] FAN Qinshan, Guan Fei, Zhao Kunmin et al. Mechanism analysis and dynamic simulation of ice-covered wire dancing [J]. Journal of Tsinghua University: Natural Science Edition, 1995 (2): 34-40.

[19] ZHU Kuanjun, Liu Bin, Liu Chaoqun, et al. Anti-dancing research of UHV transmission lines [J]. High Voltage Engineering, 2007, 33 (11): 12-20.

[20] JIN Xidong. Genetic Algorithm and Its Application [D]. Southwest Jiaotong University, 1996.

[21] LI Xiaofeng, Xu Yiping, Wang Yinqing, et al. Establishment and Application of BP Artificial Neural Network Adaptive Learning Algorithm [J]. Systems Engineering - Theory \& Practice, 2004, 24 (5): 1-8. 